\title{
Hormona de crecimiento en sangre de papel filtro para el diagnóstico de deficiencia de hormona de crecimiento
}

\author{
Growth hormone of dried blood spot for the diagnosis of growth \\ hormone deficiency \\ Domínguez-Menéndez G. ${ }^{a}$, Cifuentes L. ${ }^{\mathrm{a}, \mathrm{b}}$, González C. ${ }^{\mathrm{c}, \mathrm{e}}$, Lagos M. ${ }^{\mathrm{a}, \mathrm{d}}$, \\ Quiroga T. ${ }^{\mathrm{a}, \mathrm{d}}$, Rumié H. ${ }^{\mathrm{a} e \mathrm{e}}$, Torres C. ${ }^{\mathrm{f}}$, Martínez-Aguayo A. ${ }^{\mathrm{a}, \mathrm{e}}$
}

aPontificia Universidad Católica de Chile

bPrograma de Salud basada en Evidencia

'Tecnólogo Médico

¿División de Laboratorios Clínicos

eSección de Endocrinología, División de Pediatría, Escuela de Medicina

fEndocrinología Pediátrica, Hospital Guillermo Grant Benavente, Concepción, Chile

Recibido el 28 de marzo de 2018; aceptado el 5 de noviembre de 2018

\begin{abstract}
Resumen
Introducción: El diagnóstico de deficiencia de hormona de crecimiento (DHC) es difícil de establecer, y se puede asociar a serias complicaciones, especialmente en el período neonatal. La prueba de estímulo de secreción de hormona de crecimiento (HC) se considera de elección para el diagnóstico, pero presenta complicaciones metodológicas y se asocia a efectos adversos. Los neonatos presentan aumento de la secreción de HC de forma fisiológica, siendo una ventana diagnóstica. Objetivo: Evaluar si la muestra de sangre en papel filtro tomada en el período neonatal, en contexto del tamizaje neonatal de hipotiroidismo congénito y fenilcetonuria, permite diferenciar pacientes con DHC, de los que no la presentan. Pacientes y Método: Estudio de casos y controles mediante determinación de concentración de HC en sangre de papel filtro extraída en período neonatal, comparando controles con DHC con casos con deficiencia descartada. Se realizó extracción de la muestra del papel filtro, obteniendo dos discos de 0,125 pulgada por cada uno de los pacientes desde el centro de la mancha de sangre del papel, para un ELISA de HC humana altamente sensible basado en el uso de anticuerpos policlonales dirigidos contra la $\mathrm{HC}$ humana recombinante de $22 \mathrm{kDa}$ de peso molecular. Resultados: Se obtuvo un total de 7 casos de DHC y 10 controles. La mediana de concentración de HC de papel filtro en los casos es 2,0 ng/ml (Rango intercuartil 3,6 ng/ml) y controles 2,05 ng/mL (RIC 2,0 $\mathrm{ng} / \mathrm{ml}$ ), U de Mann-Withney 30,5 ( $\mathrm{p}=0,68$ ). Los dos casos con deficiencia de hormonas hipofisarias múltiples (DHHM) presentan concentraciones menores a $1 \mathrm{ng} / \mathrm{ml}$. Conclusión: La muestra de papel filtro no permitió diferenciar a los pacientes con DHC de los casos controles, aunque los casos con DHHM presentaron concentraciones mucho menores, en comparación a la deficiencia de hormona de crecimiento aislada (DHCA).
\end{abstract}

Palabras clave: Déficit hormona de crecimiento; glándula pituitaria; muestra de sangre en papel filtro; enanismo

Correspondencia:

Alejandro Martínez Aguayo

alemarti@med.puc.cl 


\begin{abstract}
Introduction: The diagnosis of growth hormone deficiency (GHD) is difficult to determine, and could be associated with severe complications, especially in the neonatal period. The stimulation test of growth hormone $(\mathrm{GH})$ secretion is considered the gold standard for diagnosis, but it has methodological complications and is associated with adverse effects. Neonates present physiological increased secretion of $\mathrm{GH}$, representing a diagnostic window. Objective: To evaluate if the dried blood spot on filter paper obtained in the neonatal period, as part of a neonatal screening for congenital hypothyroidism and phenylketonuria, allows differentiating patients with GHD from those who do not have it. Patients and Method: Study of cases and controls by measuring the GH concentration in dried blood spot on filter paper obtained in the neonatal period, comparing controls with GHD with cases with discarded deficiency. The sample was extracted from the filter paper, obtaining two 0.125 inch discs per each patient from the center of the blood spot on the paper, for a highly sensitive ELISA assay for human GH based on the use of polyclonal antibodies against $22 \mathrm{kDa}$ recombinant human GH. Results: Seven cases of GHD and ten controls were obtained. The median GH concentration of the dried blood spot in the cases is $2.0 \mathrm{ng} / \mathrm{ml}$ (Interquartile range $3.6 \mathrm{ng} / \mathrm{ml}$ ) and $2.05 \mathrm{ng} / \mathrm{ml}$ (Interquartile range $2.0 \mathrm{ng} / \mathrm{ml}$ ) in the controls, Mann-Whitney U test $30.5(\mathrm{p}=0.68)$. The two cases with multiple pituitary-hormone deficiency (MPHD) present concentrations lower than $1 \mathrm{ng} / \mathrm{ml}$. Conclusion: The dried blood spot sample did not differentiate GHD patients from control cases, although MPHD cases present much lower concentrations compared to isolated growth hormone deficiency (IGHD).
\end{abstract}

\section{Keywords:}

Growth hormone deficiency; pituitary gland; dried blood spot testing; dwarfism

\section{Introducción}

La deficiencia de hormona de crecimiento (DHC) es una causa poco frecuente de talla baja, pero puede asociarse a alteraciones metabólicas graves, dentro de ellas la hipoglicemia, especialmente en el período neonatal. Se estima una prevalencia de 1 en 4.000 a 1 en $10.000^{1}$. El diagnóstico de DHC en la infancia y la adolescencia presenta múltiples controversias. Los métodos de diagnóstico utilizan elementos clínicos y auxológicos, pruebas bioquímicas del eje de la hormona de crecimiento y el factor de crecimiento insulino simil tipo 1 (IGF-1, por sus siglas en inglés), elementos radiológicos y en algunos casos incluyen estudios genéticos. Para la confirmación del diagnóstico se utilizan distintas pruebas de estímulo farmacológicas (clonidina, arginina, levodopa, glucagón, entre otras) y fisiológicas (prueba de ejercicio y determinación de hormona de crecimiento durante el sueño) para la secreción de hormona de crecimiento (HC). Existen distintos protocolos para el estudio de la producción de hormona de crecimiento mediante fármacos que estimulan su secreción. Las pruebas de estímulo no están estandarizadas, y muchas de ellas se asocian a efectos adversos, por lo que en algunos centros están contraindicadas en niños menores de 2 años. Por lo tanto, es necesario encontrar nuevos métodos diagnósticos más estandarizados y confiables.

Actualmente no contamos con métodos más específicos y reproducibles para realizar el diagnóstico definitivo de DHC, especialmente en los pacientes lactantes y preescolares. Se han buscado métodos alter- nativos para realizar el diagnóstico en estos pacientes, entre ellos el uso de muestras de papel filtro tomadas para el tamizaje neonatal de hipotiroidismo congénito y fenilcetonuria. Esto se fundamenta en base a que en los primos días de vida hay una secreción aumentada de HC de forma espontánea ${ }^{3}$, siendo un período de ventana de diagnóstico de la suficiencia de producción de $\mathrm{HC}$, concentración que se mantiene aumentada por mayor tiempo en los recién nacidos de pretérmino. Los casos con DHC deberían presentar una secreción disminucida de $\mathrm{HC}$ en este período, no produciendo el aumento fisiológico de $\mathrm{HC}$ de todos los recién nacidos. Se ha planteado determinar la concentración HC en la muestra de papel filtro neonatal como un elemento más en el estudio de la función del eje somatotropo. Binder et al. ${ }^{4}$ demostraron la utilidad de este método en pacientes con DHHM, no así en pacientes con deficiencia de HC aislada (DHCA).

El objetivo de nuestro trabajo es determinar si la muestra de sangre de papel filtro extraída en el período neonatal permite discriminar a los pacientes que tienen deficiencia congénita de hormona de crecimiento, tanto aislada como asociada a deficiencia de otras hormonas hipofisarias, de los pacientes a los que se les ha descartado ésta patología.

\section{Pacientes y Método}

\section{Sujetos y controles}

Se realizó un estudio de casos y controles. Como casos se seleccionaron pacientes con diagnóstico de 
DHC, nacidos en la Red UC-Christus entre los años 2010 y 2017, basados en al menos una prueba de estímulo alterada de secreción de HC; con una concentración plasmática menor o igual a 7,0 ng/ml (punto de corte utilizado como referencia en la guía del Ministerio de Salud de Chile para el tratamiento de deficiencia de HC, no publicado $)^{1}$, asociados a criterios auxológicos (longitud o talla $<-2 \mathrm{DE}$ ) y/o perdida de velocidad de crecimiento $(<-1 \mathrm{DE})$, estudios bioquímicos y radiológicos altamente sugerentes de deficiencia de hormona de crecimiento (hipoglicemia, ictericia neonatal, micropene, alteración anatómica hipofisaria, IGF-1 e IGFBP-3 disminuida, HC disminuida en muestra crítica tomada en hipoglicemia $(<15,0 \mathrm{ng} / \mathrm{ml}$ en periodo de recién nacido; o $<7,0 \mathrm{ng} / \mathrm{ml}$ fuera del periodo de recién nacido).

Como controles se seleccionaron a pacientes con talla baja y con una prueba de estímulo para $\mathrm{HC}$ con un resultado $>7,0 \mathrm{ng} / \mathrm{ml}$.

Los candidatos fueron identificados mediante la revisión de la base de datos de las fichas clínicas, y mediante contacto directo con los médicos endocrinólogos pediatras en la red de salud UC-Christus. Se solicitó consentimiento informado a los padres o tutores legales de cada paciente, con este firmado se accedió a la ficha clínica para obtener datos clínicos y de laboratorio correspondientes para cada uno de ellos. Se incluyeron a todos los niños que se encontraran en control en nuestro centro. Se excluyeron a todos los que no contaran con la muestra de sangre de papel filtro o sin información clínica y de laboratorio necesaria para clasificarlos como DHC. Se recopilaron datos incluyendo fecha de nacimiento, edad actual (tiempo de conservación del papel filtro), diagnóstico, sexo, edad gestacional, peso de nacimiento, elementos clínicos sugerentes de deficiencia de hormona de crecimiento, pruebas de laboratorio del eje somatotropo, prueba de estímulo de secreción de HC, edad al diagnóstico y compromiso de otros ejes hipofisarios.

\section{Condiciones de toma de la muestra en papel filtro y almacenamiento}

Ambos grupos debían contar con muestra de sangre en papel filtro tomada en el período neonatal, almacenada en el laboratorio clínico de nuestro centro. Se analizaron muestras de sangre de papel filtros conservadas a temperatura ambiente ${ }^{5,6}$. Se consideró como una probable variable confundente la antigüedad del papel filtro, que fue definida como el periodo de conservación comprendido desde la toma de muestra hasta el análisis realizado en nuestro estudio.

\section{Ensayo y técnica de laboratorio}

Se realizó extracción de la muestra del papel filtro, obteniendo dos discos de 0,125 pulgada por cada uno de los pacientes desde el centro de la mancha de sangre del papel, mediante el método indicado por el fabricante del papel filtro (Whatman, Reino Unido). Se diluyó la muestra obtenida con buffer comercialmente disponible de dilución específico para la prueba de ELISA de HC humana, entregado por el fabricante. El contenido de la $\mathrm{HC}$ en el eluído fue medido por un método de ELISA altamente sensible (Mediagnost, Reutlingen, Germany). El ensayo está basado en el uso de anticuerpos policlonales dirigidos contra la $\mathrm{HC} \mathrm{hu}$ mana recombinante de $22 \mathrm{kDa}$ de peso molecular. El ensayo está calibrado contra el Estándar Internacional 98/574. Los resultados obtenidos fueron expresados en $\mathrm{ng} / \mathrm{ml}$. Las determinaciones fueron realizadas en duplicado. Se realizó el análisis con todas las muestras disponibles.

\section{Estadística}

Los datos recolectados fueron tabulados en $\mathrm{Mi}$ crosoft Excel ${ }^{\circledR}$. Para el análisis estadístico se utilizaron los softwares SPSS ${ }^{\circledR}$ y Prism ${ }^{\circledR}$ GraphPad. Se utilizó la prueba de Mann-Whitney para el análisis, informando mediana y rango intercuartil. Para correlación se utilizó prueba de Spearman. Se consideró significativo un valor $\mathrm{p}<0,05$.

\section{Ética}

El estudio fue aprobado por el comité de ética de la Facultad de Medicina de la Pontificia Universidad Católica de Chile, número de proyecto 16-121. A todos los padres o representantes legales se les solicitó firma de una carta de consentimiento informado.

\section{Resultados}

\section{Características de la muestra}

Se reclutaron un total de 20 sujetos durante el periodo analizado, diez con deficiencia de hormona de crecimiento, de los cuales sólo siete contaban con muestra de sangre de papel filtro del período neonatal almacenadas en el laboratorio de la red UC-Christus (tabla 1), y diez sujetos controles.

En la figura 1 se comparan las características del periodo de recién nacido de los sujetos con DHC y los controles, respectivamente. Ambos grupos fueron similares en edad gestacional (figura 1A), puntaje Z del peso de nacimiento (figura $1 \mathrm{~B}$ ) y puntaje $\mathrm{Z}$ de la longitud de nacimiento (figura 1C).

\section{Resultados de $\mathrm{HC}$ en muestras de papel filtro}

En los sujetos con diagnóstico de DHC, las concentraciones de HC fluctuaron entre un mínimo de 0,2 a 7,1 ng/mL; comparados con los sujetos sin deficiencia de HC donde las concentraciones de HC fluctua- 
Tabla 1. Descripción de casos

\begin{tabular}{|c|c|c|c|c|c|}
\hline & Sexo & $\begin{array}{l}\text { Edad al diagnóstico } \\
\text { (meses) }\end{array}$ & Diagnóstico & $\begin{array}{l}\text { Edad toma de muestra } \\
\text { (días) }\end{array}$ & $\begin{array}{l}\text { HC diagnóstico } \\
(\mathrm{pg} / \mathrm{mL})\end{array}$ \\
\hline \multicolumn{6}{|l|}{ Casos } \\
\hline Caso 1 & M & 0 & $\mathrm{DMHH}$ & 11 & 0,05 \\
\hline Caso 2 & $\mathrm{H}$ & 0 & DHCA & 15 & 5,65 \\
\hline Caso 3 & M & 0 & DHCA & 7 & 4,06 \\
\hline Caso 4 & $\mathrm{H}$ & 18 & DHCA & 2 & 3,20 \\
\hline Caso 5 & $\mathrm{H}$ & 0 & DHCA & 2 & 2,07 \\
\hline Caso 6 & $\mathrm{H}$ & 8 & DHCA & 2 & 7,00 \\
\hline Caso 7 & $\mathrm{H}$ & 44 & $\mathrm{DMHH}$ & 2 & 6,00 \\
\hline \multicolumn{6}{|l|}{ Controles } \\
\hline Control 1 & M & 16 & Talla baja & 8 & 11,20 \\
\hline Control 2 & M & 39 & Talla baja & 7 & 8,05 \\
\hline Control 3 & M & 65 & Talla baja & 2 & 12,10 \\
\hline Control 4 & $\mathrm{H}$ & 8 & Talla baja & 9 & 20,80 \\
\hline Control 5 & M & 79 & Talla baja & 3 & 12,30 \\
\hline Control 6 & $\mathrm{H}$ & 26 & Talla baja & 8 & 18,30 \\
\hline Control 7 & M & 65 & Talla baja & 2 & 10,70 \\
\hline Control 8 & M & 67 & Talla baja & 1 & 11,30 \\
\hline Control 9 & M & 20 & Talla baja & 2 & 9,54 \\
\hline Control 10 & $\mathrm{H}$ & 61 & Talla baja & 3 & 19,10 \\
\hline
\end{tabular}

HC: hormona de crecimiento, M: mujer, H: hombre, DHHM:Deficiencia de Hormonas Hipofisarias Múltiples; DHCA: deficiencia de hormona de crecimiento aislada.

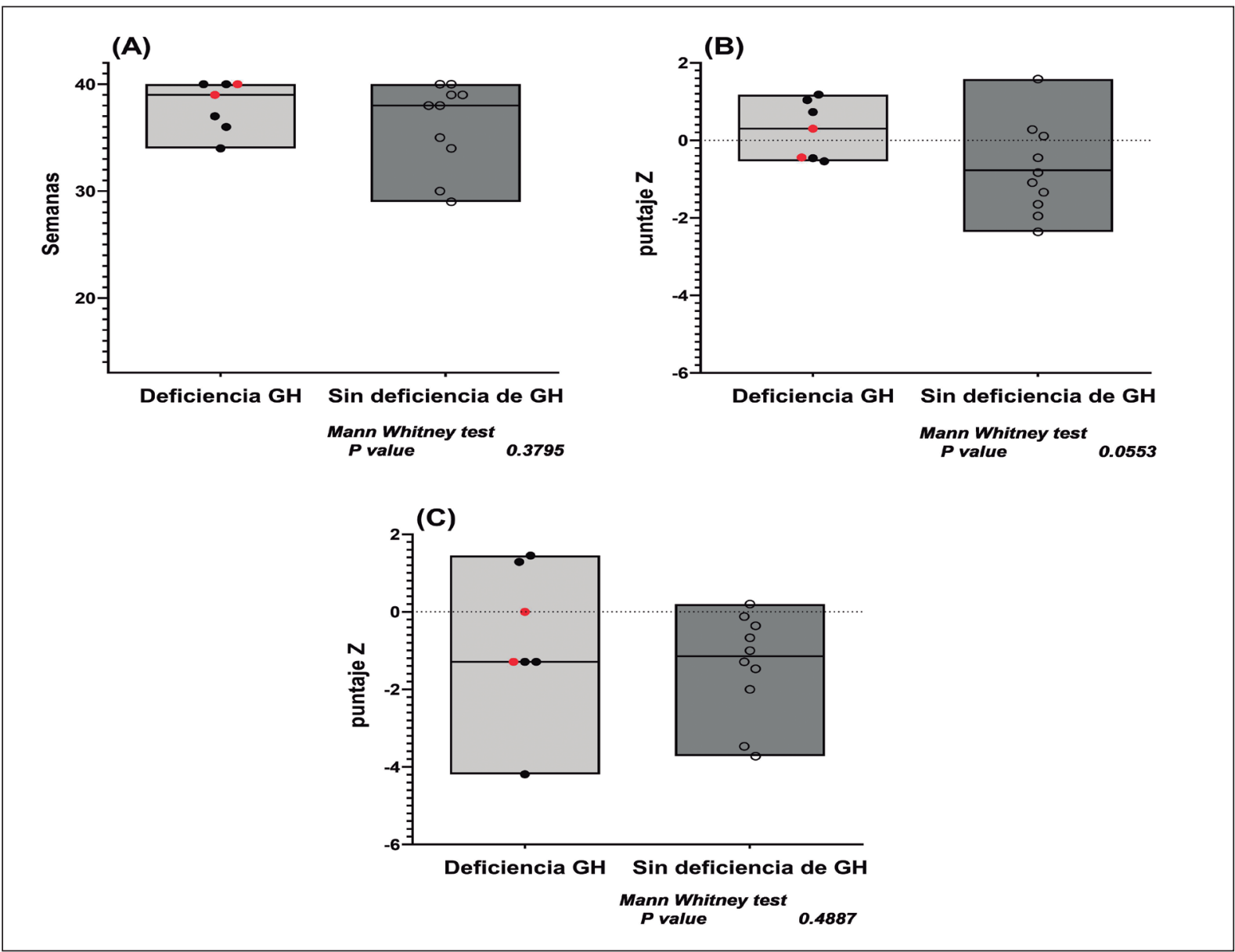

Figura 1. (A) Edad gestacional, (B) Peso de nacimiento, (C) Talla de crecimiento. Círculos rojos: deficiencia múltiple de hormonas hipofisarias. Círculos negros: deficiencia aislada de hormona de crecimiento. HC: Hormona de Crecimiento. 
(A)

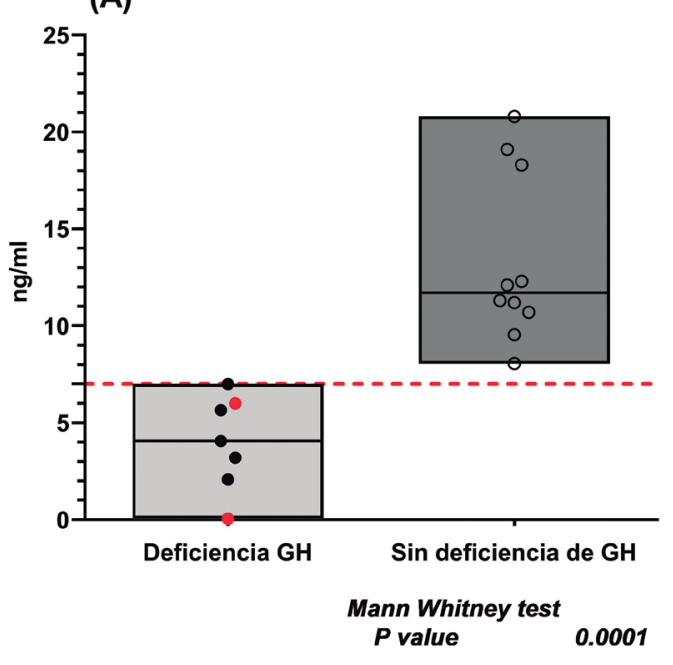

(B)

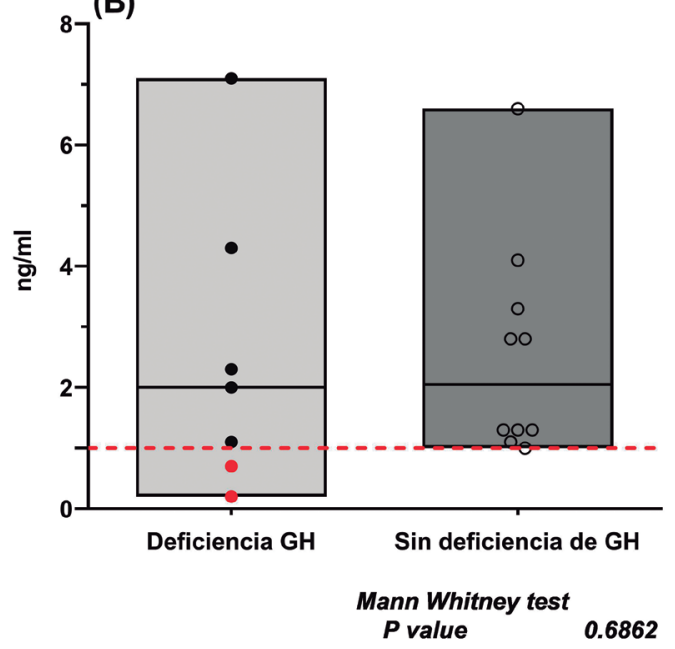

Figura 2. (A) HC al diagnóstico; (B) HC en muestra de papel filtro.

ron entre un mínimo de 1,0 a 6,6 ng/mL. Si bien no se observaron diferencias entre las medianas de la concentración de HC entre ambos grupos, es importante destacar que los dos sujetos con DMHH presentan una concentración bajo el límite inferior observado en los sujetos sin deficiencia (menor a 1,0 ng/mL) (figura $2 \mathrm{~B}$ ).

La antigüedad del papel filtro tuvo una mediana de 18 meses ( $\mathrm{RIC}=3-52$ meses) en los casos y 67 meses para los controles ( $\mathrm{RIC}=56,5-83,75$ meses); MannWhitney $\mathrm{U}=3,0 ; \mathrm{P}=0,02$ bilateral. No hubo asociación entre la antigüedad de papel filtro y el resultado de la concentración de hormona de crecimiento en ésta muestra (Rho de Spearman $=-0,1 ; \mathrm{p}=0,704$; figura 3).

\section{Discusión}

Los sujetos con deficiencia de hormonas hipofisarias múltiples tienen concentraciones de $\mathrm{HC}$ en papel filtro neonatal significativamente más bajas que las observadas en los con DHCA o sin deficiencia. La determinación de HC en papel filtro almacenada a temperatura ambiente ${ }^{5,7}$ no fue útil para distinguir entre los sujetos con deficiencia aislada o sin deficiencia.

Binder et al. ${ }^{7,8}$ demostraron la factibilidad de determinar $\mathrm{HC}$ en sangre de papel filtro, validando los ensayos de laboratorio para su análisis, pero en éstos casos el almacenamiento se realizó de forma refrigerada, a $6^{\circ}$ Celsius. En nuestro centro, el almacenamiento de las muestras de papel filtro se realiza a temperatura ambiente, en un lugar protegido de la humedad y la luz solar. Como vemos en los resultados, no existe una

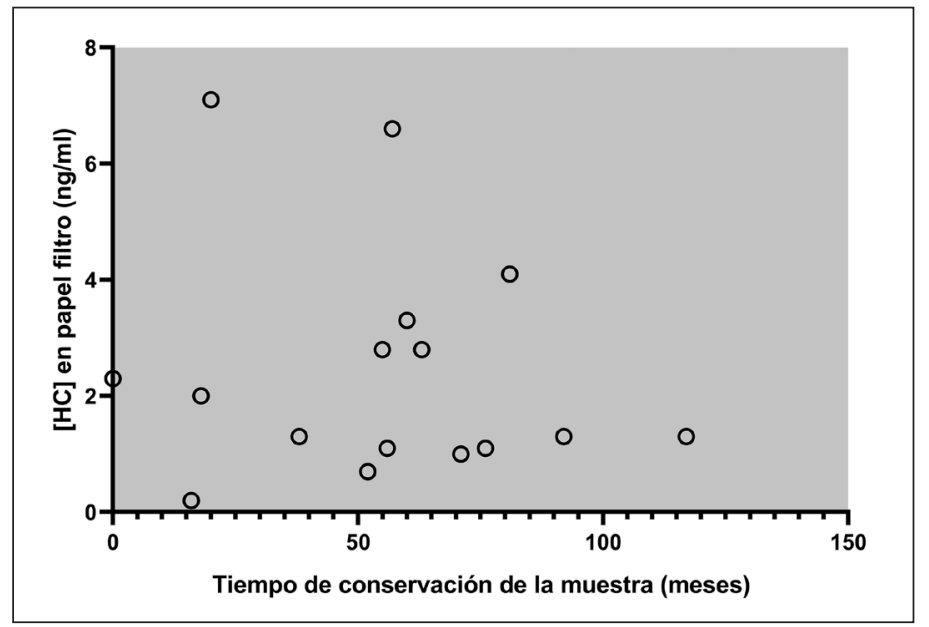

Figura 3. Correlación tiempo de conservación de muestra de papel filtro y concentración de hormona de crecimiento ([HC]) en sangre de papel filtro.

correlación directa entre el tiempo de almacenamiento de las muestras en estas condiciones, con los resultados obtenidos en la concentración de HC.

A partir de nuestros resultados podemos observar que no existe una diferencia significativa entre la concentración de $\mathrm{HC}$ obtenida de papel filtro de los pacientes que presentan un diagnóstico de DHCA y los sujetos controles.

Los dos sujetos con DHHM tuvieron concentraciones de $\mathrm{HC}$ menor al rango mínimo observado tanto en los sujetos con DHCA como los controles, con una concentración de $\mathrm{HC}$ en papel filtro $<1 \mathrm{ng} / \mathrm{ml}$. Estos resultados son concordantes con lo expuesto en un es- 
tudio previo ${ }^{7}$. Cabe destacar que uno de los pacientes con DHHM fue diagnosticado a la edad de 44 meses, con todas las repercusiones que esto podría tener en el mayor riesgo de hipoglicemias y compromiso del desarrollo neuronal.

El Caso 1 fue diagnosticado en el período neonatal en contexto de una muestra crítica por presentar hipoglicemias grave (glicemia $17 \mathrm{mg} / \mathrm{dl}$ ) y la presencia de malformaciones craneofaciales. El estudio con Resonancia Magnética muestra aplasia hipofisaria. Por otro lado, el Caso 7 fue diagnosticado a los 44 meses de edad, estudio fue realizado por presentar hipoglicemias cetósicas a los 3 años de edad asociado a convulsión, con glicemia de $18 \mathrm{mg} / \mathrm{dl}$. Se completa estudio con test de estímulo de secreción de HC con clonidina, mostrando deficiencia de HC.

Los pacientes con DHHM constituyen un espectro clínico amplio, donde se han descrito fenotipos simples, sin alteraciones anatómicas cerebrales y que corresponden a variantes patogénicas de los genes tardíos en la formación de la hipófisis. Por otro lado, hay sujetos con DHHM con un fenotipo complejo, secundario a variantes patogénicas de genes involucradas en el desarrollo temprano del cerebro, como es el caso de la displasia septo-ótica, holoprosencenfalia y otros. Los pacientes con DHHM suelen tener hipoglicemias severas por la ausencia de cortisol y HC, dos hormonas fundamentales para mantener la homeostasis de la glucosa. No realizar el diagnóstico en forma oportuna en el periodo neonatal podría asociarse a secuelas neurológicas irreversibles por hipoglicemias a repetición.

El Ministerio de Salud sugiere que las muestras de papel filtro del tamizaje neonantal se almacenen de preferencia de forma refrigerada, pero pueden ser almacenados a temperatura ambiente, pero no hay claridad del máximo tiempo que pueden ser almacenadas de esta forma9. Sin embargo, el almacenamiento a temperatura ambiente de las muestras pareciera afectar directamente el resultado obtenido en la prueba de laboratorio, disminuyendo la concentración de hormona de crecimiento en todas las muestras. Esto podría explicar por qué los resultados reportados previamente en la literatura, donde los papeles filtros son almacenados a $6^{\circ} \mathrm{C}$, son completamente diferentes a los nuestros.

Como ya fue mencionado en otros reportes, el almacenamiento de las muestras de papel filtro refrigeradas a $6^{\circ} \mathrm{C}$, la concentración detectada en el papel filtro disminuía en menor magnitud ${ }^{8}$. Cabe destacar que los pacientes controles, en los que se esperaría una concentración mayor de HC, tienen papeles filtros almacenados por un mayor tiempo, lo que podría incidir directamente en los resultados obtenidos en nuestra investigación.

Se requiere mayor evaluación de la viabilidad de la muestra de sangre de papel filtro almacenada por largos periodos de tiempo para ser utilizado como método diagnóstico de DHC u otras patologías, considerando especialmente el efecto que tiene las condiciones de almacenamiento sobre la estabilidad de la muestra.

La muestra de sangre en papel filtro es fácil de obtener, con una punción mínimamente invasiva, de fácil acceso, requiriendo una cantidad limitada de sangre, permitiendo ser realizada incluso en los recién nacidos más pequeños ${ }^{5}$. La determinación de $\mathrm{HC}$ en sangre almacenada en papel filtro, podría ser útil en identificar aquellos casos severos de DHC o con DHHM. Especialmente en lactantes y preescolares donde en ausencia de muestra crítica en hipoglicemia, realizar una prueba de estímulo farmacológico para liberar hormona de crecimiento podría tener reparos. Se requiere un nuevo estudio con papel filtro almacenado en forma refrigerada y no a temperatura ambiente, para validar esta hipótesis.

Los resultados de nuestro estudio pueden estar limitados dado el insuficiente tamaño de la muestra, ya que tenemos un número limitado de personas en control en nuestro centro que tenían su papel filtro disponible. Además, no se logró acceder a las muestras disponibles en otros centros.

Nosotros sugerimos cambiar el almacenamiento actual del papel filtro con sangre obtenida en el periodo de recién nacido, por lo que queremos proponer que sea refrigerada y guardada en bolsas individuales para evitar humedad, por lo menos a $6^{\circ} \mathrm{C}$ y por un periodo de 5 años.

La determinación de HC y de otras hormonas, como cortisol, de la muestra en papel filtro realizada durante el tamizaje neonatal podría ser útil en aquellos pacientes en los cuales no se dispone de muestra crítica durante su hipoglicemia y así poder tener un elemento más que apoye el diagnóstico de DHHM que puede conllevar riesgo vital.

\section{Responsabilidades Éticas}

Protección de personas y animales: Los autores declaran que los procedimientos seguidos se conformaron a las normas éticas del comité de experimentación humana responsable y de acuerdo con la Asociación Médica Mundial y la Declaración de Helsinki.

Confidencialidad de los datos: Los autores declaran que han seguido los protocolos de su centro de trabajo sobre la publicación de datos de pacientes.

Derecho a la privacidad y consentimiento informado: Los autores han obtenido el consentimiento informado de los pacientes y/o sujetos referidos en el 
artículo. Este documento obra en poder del autor de correspondencia.

\section{Conflicto de intereses}

Los autores declaran no tener conflicto de intereses.

\section{Agradecimientos}

Agradecemos la colaboración de nuestros pacientes, y la excelente disposición de la Sra. Rosario Muñoz, técnico paramédico de la Sala Metabólica de la Red Chritus-UC.

\section{Referencias}

1. Stanley T. Diagnosis of growth hormone deficiency in childhood. Curr Opin Endocrinol Diabetes Obes. 2012;19:47-52.

2. Growth Hormone Research Society. Consensus Guidelines for the Diagnosis and Treatment of Growth Hormone (HC) Deficiency in Childhood and Adolescence: Summary Statement of the HC Research Society. J Clin Endocrinol Metab. 2000; 85:3990-3.

3. Cornblath M, Parker M, Reisner S, Forbes A, DauHCaday W. Secretion and Metabolismo of Growth Hormone in Premature and Full-Term infants. J Clin Endocrinol Metab. 1965;25: 209-18.

4. Langkamp M, Weber K, Ranke MB. Human growth hormone measurement by means of sensitive ELISA of whole blood spots on filter paper. Growth Horm
IGF Res. 2008:18:526-532.

5. Enderle Y, Foerster K, Burhenne J. Clinical feasibility of dried blood spots: Analytics, validation, and applications. J Pharm Biomed Anal. 2016;130: 231-43.

6. Sharma A, Jaiswal S, Shukla M, Lal J. Dried blood spots: Concepts, present status, and future perspectives in bioanalysis. Drug Test Anal. 2014;6:399414.

7. Binder $\mathrm{G}$, Weidenkeller M, Blumenstock G, Langkamp M, Weber K, Franz AR. Rational approach to the diagnosis of severe growth hormone deficiency in the newborn. J Clin Endocrinol Metab. 2010;95:2219-26. Growth Horm IGF Res. 2008; 18:526-32.

8. Binder G, Hettmann S, Weber K, Kohlmüller D, Schweizer R. Analysis of the $\mathrm{HC}$ content within archived dried blood spots of newborn screening cards from children diagnosed with grwoth hormone deficiency after the neonatal period. Growth Horm IGF Res. 2011;21:314-7.

9. Cornejo V. Normas para el óptimo desarrollo de programas de búsqueda masiva de Fenilquetonuria (PKU), Hipotiroidismo congénito (HC) y otros errores congénitos del metabolism. Ministerio de Salud, Chile. http:// web.minsal.cl/portal/url/item/ dd7c4cf4c184c58de040010165016b2a.pdf, última visita 05-03-2018.

10. Cornejo V. Normas para el óptimo desarrollo de programas de búsqueda masiva de Fenilquetonuria (PKU), Hipotiroidismo congénito (HC) y otros errores congénitos del metabolism. Ministerio de Salud, Chile. http:// web.minsal.cl/portal/url/item/ dd7c4cf4c184c58de040010165016b2a.pdf, última visita 05-03-2018. 